\title{
Plug-in and plug-out dispatch optimization in microgrid clusters based on flexible communication
}

\author{
Jie $\mathrm{YU}^{1}$, Ming $\mathrm{NI}^{2}$, Yiping $\mathrm{JIAO}^{3}$, Xiaolong $\mathrm{WANG}^{3}$
}

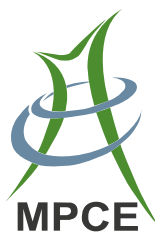

\begin{abstract}
With large-scale development of distributed generation (DG) and its potential role in microgrids, the microgrid cluster (MGC) becomes a useful control model to assist the integration of DG. Considering that microgrids in a MGC, power dispatch optimization in a MGC is difficult to achieve. In this paper, a hybrid interactive communication optimization solution (HICOS) is suggested based on flexible communication, which could be used to solve plug-in or plug-out operation states of microgrids in MGC power dispatch optimization. HICOS consists of a hierarchical architecture: the upper layer uses distributed control among multiple microgrids, with no central controller for the MGC, and the lower layer uses a central controller for each microgrid. Based on flexible communication links among microgrids, the optimal iterative information are exchanged among microgrids, thus HICOS would gradually converge to the global optimal solution. While some microgrids plug-in or plug-out, communication links will be changed, so as to unsuccessfully reach optimal solution. Differing from changeless communication links in traditional communication networks, HICOS redefines the topology of flexible communication links to meet the requirement to reach the global optimal solutions.
\end{abstract}

CrossCheck date: 5 August 2016

Received: 28 July 2015 / Accepted: 5 August 2016/Published online: 14 October 2016

(C) The Author(s) 2016. This article is published with open access at Springerlink.com

$\triangle$ Jie YU

yujie@seu.edu.cn

1 School of Electrical Engineering, Southeast University, Nanjing, China

2 NARI Technology Co. Ltd, Nanjing, China

3 School of Automation, Southeast University, Nanjing, China
Simulation studies show that HICOS could effectively reach the global optimal dispatch solution with non-MGC center. Especially, facing to microgrids plug-in or plug-out states, HICOS would also reach the global optimal solution based on refined communication link topology.

Keywords Microgrid Cluster (MGC), Plug-in/plug-out dispatch optimization, hybrid interactive communication optimization solution(HICOS), Flexible communication

\section{Introduction}

With the continuing growth of energy consumption and growing attention to environmental pollution, the value of distributed generation (DG) is becoming more and more important. Effectively managing a large amount of DG connected to the grid is a significant challenge.

Microgrids are a mechanism to meet this challenge in their role as independent control systems containing DG, loads and energy storage devices [1, 2]. Large-scale application of microgrids in distribution power grids will require efficient integration of multiple microgrids [3-5]. In this paper we propose that a Microgrid Cluster (MGC), as an aggregator of microgrids, would help to achieve optimal dispatch of DG and enhance power system reliability and power quality $[6,7]$.

Microgrids are usually capable of both islanded and gridconnected operation, and being autonomous to switch between these states, which is called as-plug-in or plug-out [8]. It is a difficult problem how to deal with microgrid plugs in and plugs out in MGC in real operating time. Chen proposed microgrid economic scheduling and developed a mathematical model [9], based on the integration of plug-in hybrid electric vehicles (PHEVs), that may be solved using a 
generic optimisation algorithm. Whereas, the problem of microgrid plug-in and plug-out in a MGC would not be solved in [9]. A solution to this problem is expected to be found on a feasible control architecture in MGCs. The following section lists control architectures used for microgrids and considers their suitability for controlling MGCs.

Microgrid control architecture can be classified into three modes: centralized, decentralized and distributed. The centralized control architecture features a control center, which sends control command to all dispatchable units (loads and generators) in the microgrid, and receives information from them. The advantage of this control mode is speedy and accurate control, addressing voltage fluctuations, frequency variation, harmonic distortion [10], and maintaining reliable supply [11]. Efficient centralized control would construct efficient and stable communication links from the control center to every unit. Microgrid plugs in and plugs out, without stable communication links with the control center, which would increase the realization difficulties of the control center.

In the decentralized control architecture, every unit controller works locally, without a control center [12] and without direct communication with other units. The local controllers can be appropriately adjusted when the parameters or the topology of the system changes, and moreover, it is not difficult for this architecture to offer additional degrees of freedom and increased controllability for each unit [13, 14]. Ahn and Peng [15] suggested a decentralized voltage control algorithm that minimizes power losses for microgrids. Microgrid plugs in and plugs out, without communication links with other units, which would not be organized as a MGC integration.

Distributed control, based on flexible communication links between units, would coordinate local autonomy with global targets [16]. The distributed units would communicate with their neighbor units and obtain current status parameters from others according to already existing communication links [17]. A large amount of research has addressed distributed control. Zhang et al. [18] applied distributed control to a multi-agent system to realize optimal generation control and economic dispatch. Xin et al. [19] researched distributed control to coordinate the outputs of a number of photovoltaic generations. Yang et al. [20] maximize virtual power plant (VPP) profits by coordinated individual decision-making of distributed energy resources via limited communication.

Considering the microgridplugs in and plugs out, this paper proposes a hybrid hierarchical architecture for MGC control, consisting of a upper layer using distributed control and a lower layer using centralized control. In the upper layer, each microgrid in an MGC coordinates with others using distributed control based on flexible communication between microgrids, which means there is no centralized controller for the MGC. Meanwhile, the lower layer employs centralized control to manage all units within each microgrid. The advantage of hierarchical architecture lies on combining MGC global targets and each microgrid's local targets. Meanwhile, distributed optimization in the upper layer can manage microgrid plugin and plug-out.

\section{The MGC architecture based on flexible communication}

A MGC integrates a number of microgrids into a control cluster, based on flexible communication among microgrids (Fig. 1). These communication links could be realized via fibre-optic, wireless, power line carrier, and so on, which are already widely used in the distribution power grid. Therefore, a MGC would not need to rebuild communication channels among microgrid nodes, but could instead use existing communication links.

In a MGC, the upper layer performs coordination among microgrids based on communication links. Meanwhile, the lower layer indicates that each microgrid controls its own distributed generators, dispatchable loads, and energy storage units. This two-layer hierarchical structure should synthetically integrate global targets with local targets of each microgrid. In other words, the control decisions made in the upper and lower layers should interact with each other.

Based on the two-layer control architecture in a MGC, a hierarchical distributed optimization mathematic model is established in Section 3, and a method for solving this model using the hybrid interactive communication optimization solution(HICOS) between layers is presented in Section 4.

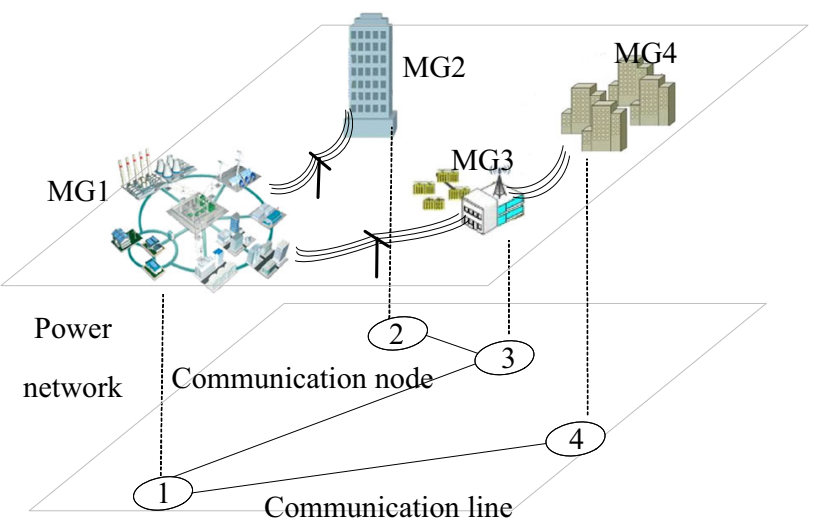

Flexible communication network based on SND

Fig. 1 Microgrid cluster control architecture based on flexible communication 


\section{Mathematic model of hierarchical hybrid optimization}

In MGC hierarchical architecture, the upper layer operates in accordance to distributed coordinated mode, without an upper control center. The aim of the upper model is to minimize the total costs of energy supply in the MGC, with the constraints of power exchange constraints and transmission lines constraints. Meanwhile, in the lower layer each microgrid manages its own distributed generators using a centralized controller. The objectives of the lower layer are to minimize costs for each microgrid with the operating constraints of distributed generations and the energy storage units.

\subsection{Upper layer distributed optimization of a MGC (ULDOM)}

The optimization model for the MGC is defined as follows.

\subsubsection{Objective function}

$\min C=\min \sum_{i=1}^{I_{\mathrm{MG}}} f_{i}\left(P_{\mathrm{ex}}^{i}\right)=\min \sum_{i=1}^{I_{\mathrm{MG}}} a_{i}\left(P_{\mathrm{ex}}^{i}\right)^{2}+b_{i} P_{\mathrm{ex}}^{i}+c_{i}$

where $C$ is the total costs of multiple microgrids; $I_{\mathrm{MG}}$ the number of microgrids controlled by distributed dispatch; $f_{i}$ the cost function of microgrid $i$, which is often a quadratic function, whose coefficients of the quadratic term, linear term and constant term are $a_{i}, b_{i}$ and $c_{i}$ respectively; $P_{\mathrm{ex}}^{i}$ the exchange power between microgrid $i$ and the main grid, where $P_{\mathrm{ex}}^{i}>0$ indicates that the main grid transfers energy to microgrid $i$; and $P_{\mathrm{ex}}^{i}<0$ means microgrid $i$ transfers energy to the main grid.

\subsubsection{Constraints}

The upper and lower bound constraints of the power exchanged between the main power grid and microgrid $i$ are:

$P_{\mathrm{ex}, \min }^{i} \leq P_{\mathrm{ex}}^{i} \leq P_{\mathrm{ex}, \max }^{i} \quad i=1,2, \ldots, I_{\mathrm{MG}}$

where $P_{\text {ex,min }}^{i}, P_{\text {ex } \max }^{i}$ are the lower and upper bounds of the exchanged power, which are decided by the transmission constraints of power lines connecting the microgrid to the main power grid.

The power demand constraint is:

$\sum_{i=1}^{I_{\mathrm{MG}}} P_{\mathrm{ex}}^{i}=P_{\mathrm{demand}}$ where $P_{\text {demand }}$ is the power generation command from the power system dispatch center, which requires the MGC to have a total power consumption of $P_{\text {demand }}$.

\subsection{Lower layer optimization of each microgrid (LLOM)}

Taking microgrid $i$ for instance, the optimization probme for each microgrid is stated as follows.

\subsubsection{Objective Function}

$$
\begin{aligned}
C_{\mathrm{F}}^{i}= & \min \sum_{j=1}^{J_{i}} C_{j}^{i}\left(P_{j}^{i}\right) \\
= & \min \left(C_{\mathrm{wind}-\mathrm{om}}^{i} P_{\mathrm{wind}}^{i}+C_{\mathrm{pv}-\mathrm{om}}^{i} P_{\mathrm{pv}}^{i}+C_{\mathrm{mt}}^{i} P_{\mathrm{mt}}^{i}+C_{\mathrm{mt}-\mathrm{om}}^{i} P_{\mathrm{mt}}^{i}\right) \\
& +C_{\mathrm{bs}-\mathrm{om}}^{i}\left(P_{\mathrm{BT}-\mathrm{dis}}^{i}+\left|P_{\mathrm{BT}-\mathrm{ch}}^{i}\right|\right)
\end{aligned}
$$

where $C_{\mathrm{F}}^{i}$ is the total cost of operating microgrid $i ; P_{i}^{j}$ the planned generation of the $j^{\text {th }}$ distributed generator in $i^{\text {th }}$ microgrid; $C_{j}^{i}\left(P_{j}^{i}\right)$ the generation cost function of generator $i$ in microgrid $i$; $J_{i}$ the number of distributed generators in microgrid $i ; P_{\text {wind }}^{i}$ the output of wind generation in microgrid $i ; C_{\text {wind-om }}^{i}$ the operation and maintenance cost factor of wind power equipment; $P_{\mathrm{pv}}^{i}$ the output of photovoltaic generation in microgrid $i ; C_{\mathrm{pv}-\mathrm{om}}^{i}$ the operation and maintenance cost factor of photovoltaic power equipment; $C_{\mathrm{mt}-\mathrm{om}}^{i}$ the operation and maintenance cost factor of microturbine generation; $P_{\mathrm{mt}}^{i}$ the output of microturbine generation in microgrid $i$; $C_{\mathrm{mt}}^{i}$ the fuel cost factor of microturbine generation; $C_{\mathrm{bs}-\mathrm{om}}^{i}$ the operation and maintenance costs factor of storage; $P_{\mathrm{BT}-\mathrm{ch}}^{i} / P_{\mathrm{BT} \text {-disch the }}^{i}$ charge/discharge power of the storage, which is positive while discharging and negative while charging.

\subsubsection{Constraints}

The power flow constraints are:

$\sum_{j=1}^{J_{i}} P_{j}^{i}-P_{\mathrm{BT}-\mathrm{ch}}^{i} \cdot \eta_{\mathrm{BT}-\mathrm{ch}}^{i}-P_{\mathrm{d}}^{i}=P_{\mathrm{ex}}^{i}$

$\sum_{j=1}^{J_{i}} P_{j}^{i}+P_{\mathrm{BT}-\mathrm{dis}}^{i} \cdot \eta_{\mathrm{BT}-\mathrm{dis}}^{i}-P_{\mathrm{d}}^{i}=P_{\mathrm{ex}}^{i}$

where $\eta_{\mathrm{BT}-\mathrm{ch}}^{i} / \eta_{\mathrm{BT}-\mathrm{dis}}^{i}$ is the charge/discharge efficiency of storage devices in microgrid $i ; P_{\mathrm{ex}}^{i}$ the exchange power of microgrid $i$ and the main grid; $P_{\mathrm{d}}^{i}$ the loads of microgrid $i$. 
The distributed generator constraints in microgrid $i$ are:

$P_{j, \min }^{i} \leq P_{j}^{i} \leq P_{j, \max }^{i} \quad j=1,2, \ldots, J_{i}$

where $P_{j \text {,min }}^{i}$ and $P_{j, \max }^{i}$ are the upper and lower bounds of distributed generator $j$.

The energy storage constraints of microgrid $i$ are:

$P_{\mathrm{BT}-\mathrm{ch}, \min }^{i} \leq P_{\mathrm{BT}-\mathrm{ch}}^{i} \leq P_{\mathrm{BT}-\mathrm{ch}, \max }^{i}$

$P_{\mathrm{BT}-\mathrm{dis}, \min }^{i} \leq P_{\mathrm{BT}-\mathrm{dis}}^{i} \leq P_{\mathrm{BT}-\mathrm{dis}, \max }^{i}$

$B_{\min }^{i} \leq B^{i} \leq B_{\max }^{i}$

where $P_{\mathrm{BT}-\mathrm{ch} \text {,min }}^{i}, P_{\mathrm{BT}-\mathrm{ch} \text {,max }}^{i}$ are the upper and lower charging power and $P_{\mathrm{BT}-\text { dis,min }}^{i}, P_{\mathrm{BT} \text {-dis,max }}^{i}$ are the upper and lower discharging power in microgrid $i ; B^{i}$ the battery storage state of charge in microgrid $i ; B_{\min }^{i}$ and $B_{\max }^{i}$ the upper and lower limits of battery storage state of charge respectively.

\section{Hybrid interactive communication optimization solution (HICOS)}

This paper proposes the hybrid interactive communication optimization solution (HICOS) method to solve the mathematic model ((1)-(10)), which integrates upper-layer distributed optimization ((1)-(3)) and lowe-layer centralized optimization ((4)-(10)).

Constraints (2) can be realized by projection operation [21], and the Lagrangian function of upper-layer optimization can be written as:

$L\left(\boldsymbol{P}_{\mathrm{ex}}\right)=\sum_{i=1}^{I_{\mathrm{MG}}} f_{i}\left(P_{\mathrm{ex}}^{i}\right)-\mu\left(\sum_{i=1}^{I_{\mathrm{MG}}} P_{\mathrm{ex}}^{i}-P_{\mathrm{demand}}\right)$

where $\mu$ is the Lagrangian multiplier of the power demand constraint (3).

The optimization problem can be solved by distributed optimization iterations based on the sub-gradient [21]:

$\boldsymbol{P}_{\mathrm{ex}}^{i}(k+1)=P R_{P_{\mathrm{ex}}^{i}}\left\{\sum_{j \in \Gamma_{i}} a_{j i} \boldsymbol{P}_{\mathrm{ex}}^{j}(k)-\alpha(k) \times \frac{\partial L}{\partial \boldsymbol{P}_{\mathrm{ex}}^{i}}\right\}$

$\boldsymbol{\mu}_{i}(k+1)=P R_{\mu_{i}}\left\{\sum_{j \in \Gamma_{i}} a_{j i} \boldsymbol{\mu}_{j}(k)-\alpha(k) \times \frac{\partial L}{\partial \boldsymbol{\mu}_{i}}\right\}$

where $\boldsymbol{P}_{\mathrm{ex}}^{i}$ and $\mu_{i}$ are the primal and dual variables stored in node $i ; \Gamma_{i}$ the neighbor node set of node $i ; \alpha(k)$ the step size at iteration $k ; L$ the Lagrangian function (11); $P R_{P_{\mathrm{ex}}^{i}}$ the projection operation towards feasible solution space determined by (2); $P R_{\boldsymbol{\mu}_{i}}$ the projection operation to area $\boldsymbol{\mu}_{i} \geq 0$ [21]; and $a_{j i}$ an element in row $j$ and column $i$ of the communication connection matrix $\boldsymbol{A}$, which denotes the communication link topology among microgrids.
If there is no communication connection between node $i$ and $j, a_{i j}$ would be zero; otherwise, $a_{i j}$ means the weight influence from node $j$ to $i$. Using HICOS to solve the distributed optimization, $\boldsymbol{A}$ should meet the requirement of a doubly-stochastic matrix. That means the sum of all $a_{i j}$ (matrix elements in the same raw $i$ or in the same column $j$ ) must be 1 [21], which can be written as

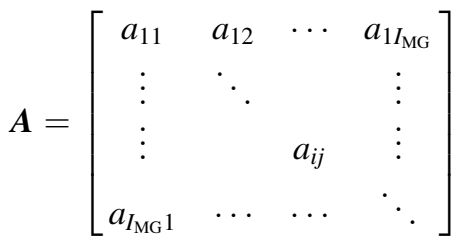

and satisfied by

$\left\{\begin{array}{l}\sum_{i=1}^{I_{\mathrm{MG}}} a_{i j}=1 \quad j=1,2, \cdots, I_{\mathrm{MG}} \\ \sum_{j=1}^{I_{\mathrm{MG}}} a_{i j}=1 \quad i=1,2, \cdots, I_{\mathrm{MG}}\end{array}\right.$

HICOS incorporates distributed optimization in the upper layer and centralized optimization in the lower layer. Meanwhile, the microgrid plugs in and plugs out could be modelled by changes in the communication connection matrix $\boldsymbol{A}$. The algorithm for HICOS is shown by the steps below:

(1) If starting iteration, set $k=0$; else $k=k+1$;

(2) Calculate iteratively $\boldsymbol{P}_{\mathrm{ex}}^{i}(k+1)$ and $\boldsymbol{\mu}_{i}(k+1)$ according to (12) and (13) after receiving information from neighbors $\boldsymbol{P}_{\mathrm{ex}}^{i}(k)$ and $\boldsymbol{\mu}_{i}(k)$ via communication links;

(3) Send $\boldsymbol{P}_{\text {ex }}^{i}(k+1)$ to the lower-layer optimisation as the power exchange constraint of microgrid $i$ (shown in (4));

(4) Solve the lower-layer control problem (shown in (4)(10)) in each microgrid via centralized optimization;

(5) Achieve the microgrid optimal results $P_{i}^{j}$, which is the planned generation output of the $j^{\text {th }}$ distributed generator in $i^{\text {th }}$ microgrid;

(6) Update $\boldsymbol{P}_{\mathrm{ex}}^{i}(k)$ as the iteration results of step $k$;

(7) Transfer $\boldsymbol{P}_{\mathrm{ex}}^{i}(k)$ to neighbor microgrids as exchange information using the communication links;

(8) If microgrid $j$ plugs in or plugs out, change the connection matrixAaccording to flexible communication;

(9) Judge whether convergence has been achieved; if not, go to (1).

Iterations continue until the termination conditions of the hybrid hierarchy optimal model is met. This kind of algorithm has been widely applied in distributed optimization problem [21, 22]. The flowchart of the iterative algorithm is shown in Fig. 2. 


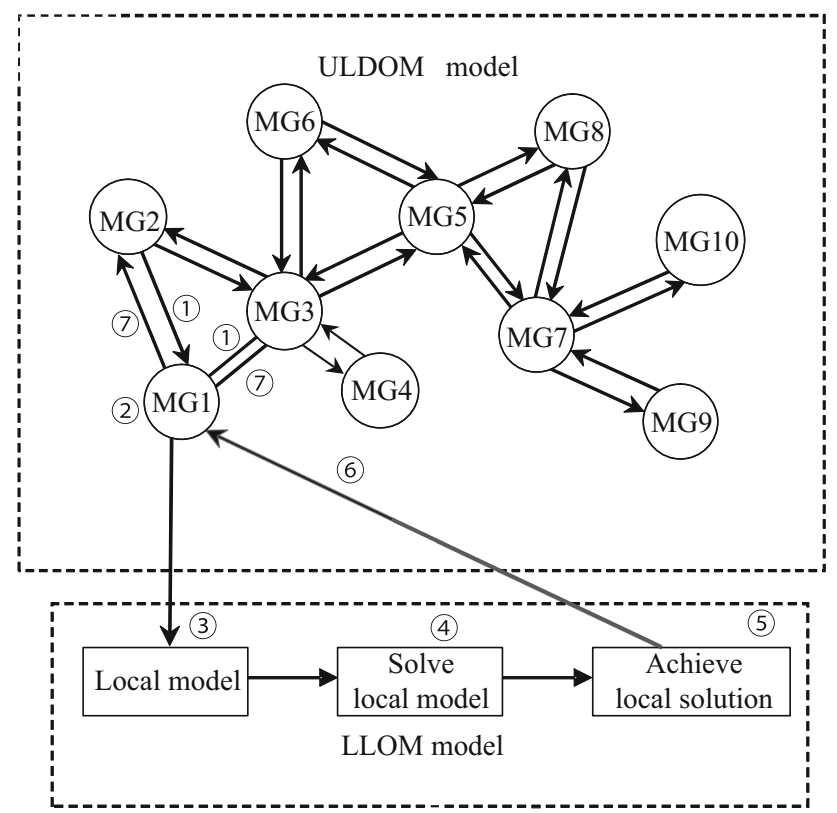

Fig. 2 Flowchart of HICOS

\section{Numerical simulations}

Taking a MGC containing 10 microgrids as an example for simulation, MGC dispatch optimization considering microgrid plug-in and plug-out is solved by HICOS as proposed by this paper. The communication topology among microgrids is represented by the connection matrix $\boldsymbol{A}$, which should be a doubly stochastic matrix [22, 23], so as to guarantee convergence of the distributed optimization algorithm.

\subsection{Case I: HICOS solution}

MGC, non-control-center, would realize multiple microgrids coordinated operation based on flexible communication. The communication links among microgrids would fully apply the existing communication channels in the distribution power grid, which includes different mode, such as fiber-optic, wireless, power line carrier, and so on. The communication topology of the simulated MGC containing 10 microgrids is shown in Fig. 3.

The connection topology matrix $\boldsymbol{A}$, denoting the communication links among microgrids, should be a doublystochastic matrix. The communication connection matrix of the MGC containing 10 microgrids is shown in (16).

$\begin{gathered}\boldsymbol{A}_{0} \\ (10 \times 10)\end{gathered}=\left[\begin{array}{cccccccccc}0.8 & 0.1 & 0.1 & 0 & 0 & 0 & 0 & 0 & 0 & 0 \\ 0.1 & 0.8 & 0.1 & 0 & 0 & 0 & 0 & 0 & 0 & 0 \\ 0.1 & 0.1 & 0.5 & 0.1 & 0.1 & 0.1 & 0 & 0 & 0 & 0 \\ 0 & 0 & 0.1 & 0.9 & 0 & 0 & 0 & 0 & 0 & 0 \\ 0 & 0 & 0.1 & 0 & 0.6 & 0.1 & 0.1 & 0.1 & 0 & 0 \\ 0 & 0 & 0.1 & 0 & 0.1 & 0.8 & 0 & 0 & 0 & 0 \\ 0 & 0 & 0 & 0 & 0.1 & 0 & 0.6 & 0.1 & 0.1 & 0.1 \\ 0 & 0 & 0 & 0 & 0.1 & 0 & 0.1 & 0.8 & 0 & 0 \\ 0 & 0 & 0 & 0 & 0 & 0 & 0.1 & 0 & 0.9 & 0 \\ 0 & 0 & 0 & 0 & 0 & 0 & 0.1 & 0 & 0 & 0.9\end{array}\right]$

In stable conditions, the MGC, organized by 10 microgirds, operates coordinately based on the communication links among microgrids. Hierarchical hybrid optimization mathematic model ((1)-(10)) is solved by the hybrid interactive communication optimization solution(HICOS) method, according to the iterative steps (shown in Fig. 2). The optimization results for the simulated MCG are illustrated in Fig. 4. Each microgrid exchanges its state information via communication links with the connection topology matrix $\boldsymbol{A}_{0}$. The iterations will ultimately converge to the globally optimal solution.

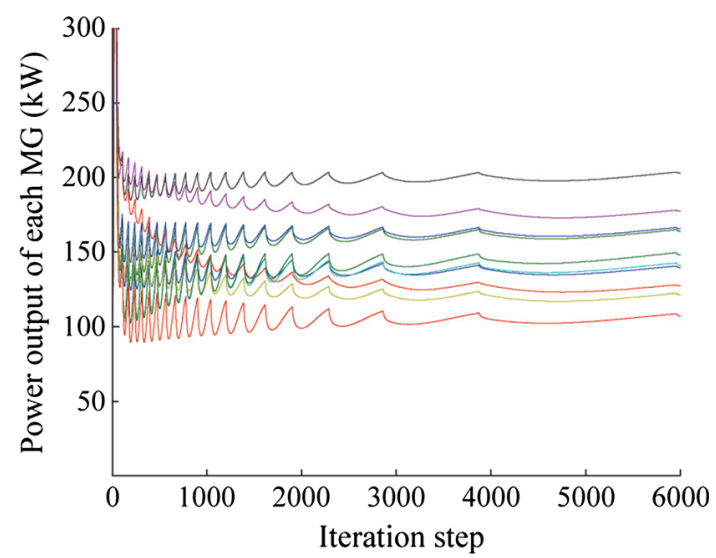

Fig. 4 Optimization results based on communication topology $\boldsymbol{A}_{0}$

Fig. 3 Communication link topology of MGC 


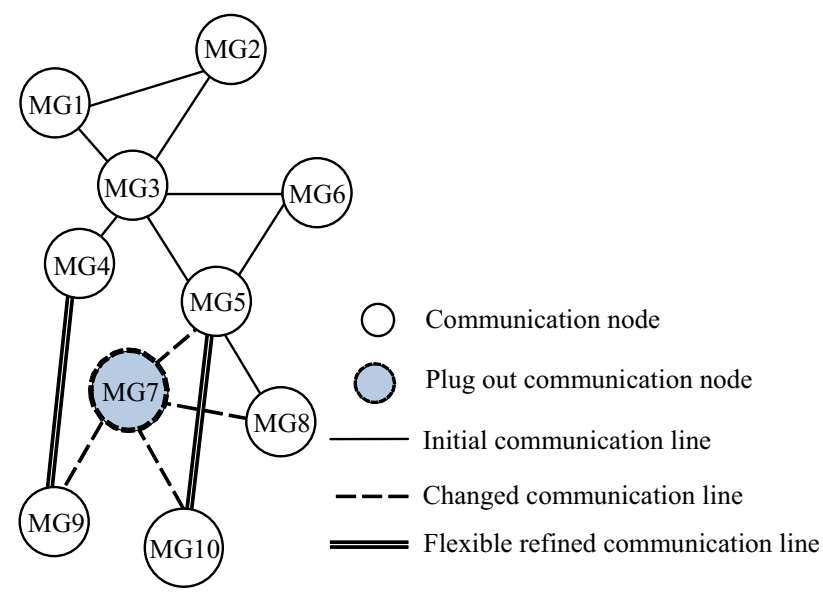

Fig. 5 Flexible communication topology when one or more microgrids plug out

\subsection{Case II: HICOS considering microgrid plugs out}

When one or more microgrids plug out from MGC, the topology of communication will undoubtedly be changed. Based on the original topology shown in Fig. 5, the communication links from the $7^{\text {th }}$ node to the $5^{\text {th }}, 8^{\text {th }}, 9^{\text {th }}$, and $10^{\text {th }}$ nodes will be dropped when microgrid $7^{\text {th }}$ plugs out. Therefore, the original connection topology matrix $\boldsymbol{A}_{0}$ would be changed into $A_{\text {plug-out }}^{\prime}$, shown in (17).

$\underset{(10 \times 10)}{A_{\text {plug-out }}^{\prime}}=\left[\begin{array}{cccccc:c:ccc}0.8 & 0.1 & 0.1 & 0 & 0 & 0 & 0 & 0 & 0 & 0 \\ 0.1 & 0.8 & 0.1 & 0 & 0 & 0 & 0 & 0 & 0 & 0 \\ 0.1 & 0.1 & 0.5 & 0.1 & 0.1 & 0.1 & 0 & 0 & 0 & 0 \\ 0 & 0 & 0.1 & 0.9 & 0 & 0 & 0 & 0 & 0 & 0 \\ 0 & 0 & 0.1 & 0 & 0.6 & 0.1 & 0 & 0.1 & 0 & 0 \\ 0 & 0 & 0.1 & 0 & 0.1 & 0.8 & 0 & 0 & 0 & 0 \\ \hdashline 0 & 0 & 0 & 0 & 0 & 0 & 0 & 0 & 0 & 0 \\ \hdashline 0 & 0 & 0 & 0 & 0.1 & 0 & 0 & 0.8 & 0 & 0 \\ 0 & 0 & 0 & 0 & 0 & 0 & 0 & 0 & 0.9 & 0 \\ 0 & 0 & 0 & 0 & 0 & 0 & 0 & 0 & 0 & 0.9\end{array}\right]$

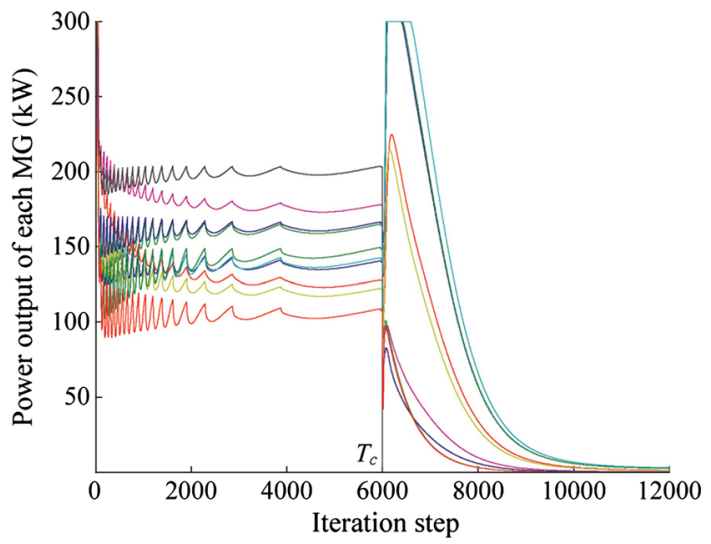

Fig. 6 Optimization results of communication topology $\boldsymbol{A}_{\text {plug-out }}^{\prime}$
After reaching optimization results based on connection topology $\boldsymbol{A}_{0}$, microgrid $7^{\text {th }}$ plugs out at the time $T_{c}$. The connection matrix $\boldsymbol{A}_{0}$ is changed into $\boldsymbol{A}_{\text {plug-out }}^{\prime}$ (17), which is no longer a stochastic matrix, so the optimization results decrease to zero, as shown in Fig. 6.

To maintain communication connectivity among microgrids in the MGC, communication links between the nodes $4^{\text {th }}$ and $9^{\text {th }}$ node $5^{\text {th }}$ and $10^{\text {th }}$ are constructed. The connection topology matrix is therefore changed to $\boldsymbol{A}_{\text {plug-out }}^{\text {modified }}$, shown in (18).

$$
\begin{gathered}
\boldsymbol{A}_{\text {plug-out }}^{\text {modified }}(9 \times 9) \\
9 \times 9
\end{gathered}=\left[\begin{array}{ccccccccc}
0.8 & 0.1 & 0.1 & 0 & 0 & 0 & 0 & 0 & 0 \\
0.1 & 0.8 & 0.1 & 0 & 0 & 0 & 0 & 0 & 0 \\
0.1 & 0.1 & 0.5 & 0.1 & 0.1 & 0.1 & 0 & 0 & 0 \\
0 & 0 & 0.1 & 0.8 & 0 & 0 & 0 & 0.1 & 0 \\
0 & 0 & 0.1 & 0 & 0.6 & 0.1 & 0.1 & 0 & 0.1 \\
0 & 0 & 0.1 & 0 & 0.1 & 0.8 & 0 & 0 & 0 \\
0 & 0 & 0 & 0 & 0.1 & 0 & 0.9 & 0 & 0 \\
0 & 0 & 0 & 0.1 & 0 & 0 & 0 & 0.9 & 0 \\
0 & 0 & 0 & 0 & 0.1 & 0 & 0 & 0 & 0.9
\end{array}\right]
$$

While microgrid $7^{\text {th }}$ plugs out at the time $T_{c}$, the connection matrix $\boldsymbol{A}_{0}$ is changed into $\boldsymbol{A}_{\text {plug-out }}^{\text {modified }}$, which is also a doublystochastic matrix. The optimization algorithm would begin converging again and would converge to a new globally optimal solution, as shown in Fig. 7.

\subsection{Case III: HICOS considering microgrid plugs in}

When some microgrids plug in, new communication links should be constructed between the new microgrids and the existing microgrids. In the simulation there are new microgrids $11^{\text {th }}$ and $12^{\text {th }}$ plug into the MGC, so the new communication node $11^{\text {th }}$ and $12^{\text {th }}$ are connected with the existing node $2^{\text {nd, }} 5^{\text {th }}$ and $6^{\text {th }}$, as shown in Fig. 8.

Then the connection matrix $\boldsymbol{A}_{0}$ would be changed according to the new communication links, maintaining the characteristics of a doubly-stochastic matrix, shown in Eq. 19.

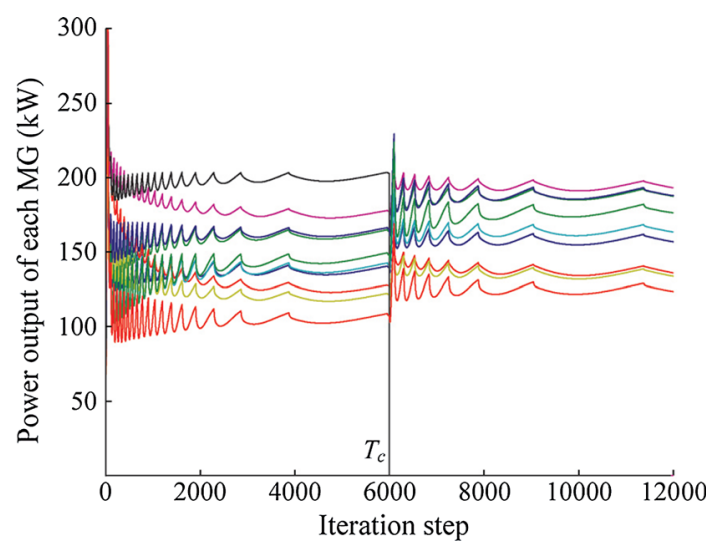

Fig. 7 Optimization results based on connection topology $\boldsymbol{A}_{\text {plug-out }}^{\text {modified }}$ 


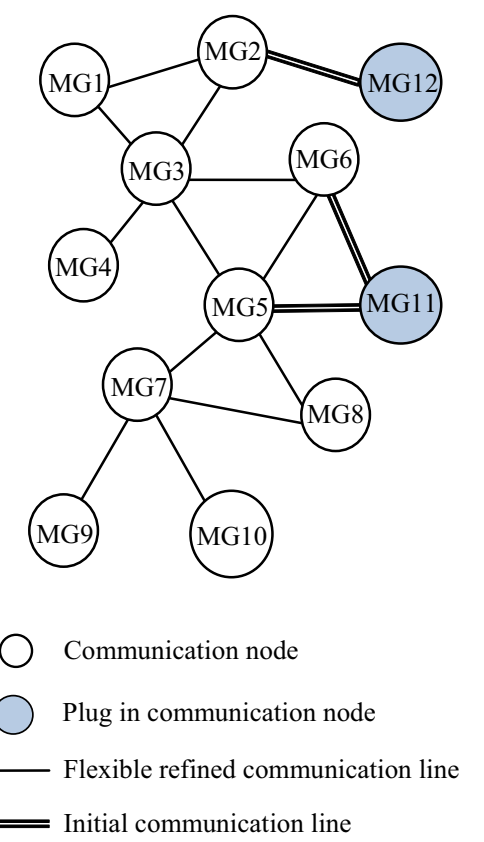

Fig. 8 Flexible communication topology when microgrids plug in

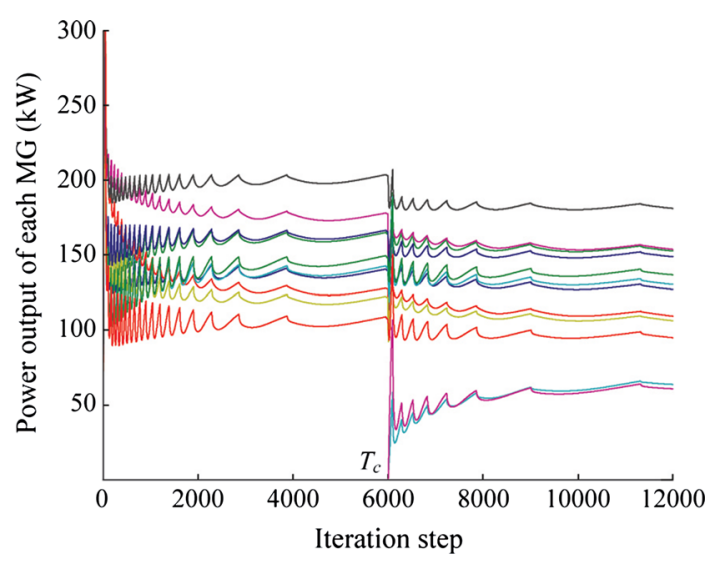

Fig. 9 Optimization results based on communication topology $A_{\text {plug-in }}$

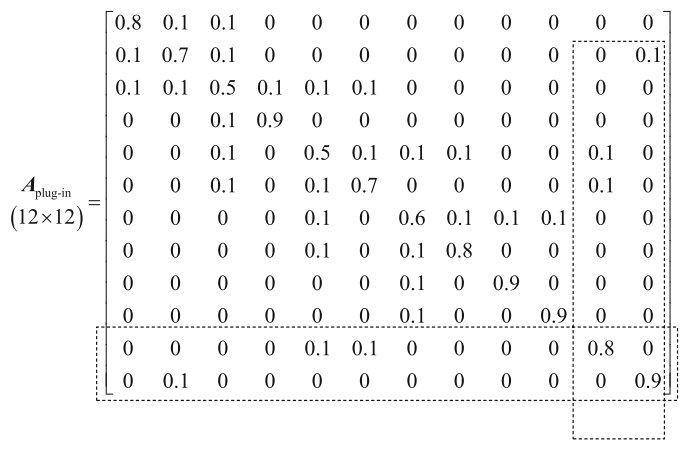

At time $T_{c}$ in the simulation, the new microgrids plug into the MGC. Based on the refreshed connection matrix $\boldsymbol{A}_{\text {plug-in }}$, the optimization results solved by HICOS are illustrated as Fig. 9.

\section{Conclusion}

To support largescale integration of microgrids into power grids, a MGC (Microgrid Cluster) with distributed control is an effective solution to consider microgrids plug in or plug out. This paper constructs a MGC hierarchical hybrid optimization model, including upper-layer distributed optimization and lower-layer centralized optimization of each microgrid. HICOS (hybrid interactive communication optimization solution) is proposed as a novel solution to this optimization problem based on the flexible communication topology. The simulation cases testify the effectiveness of HICOS. The novel optimization model and methods in this paper, accustomed to plug-in and plug-out dispatch optimization in MGC based on flexible communication, are suitable for challenging and constructive experiments that demonstration practical operation of microgrids in a MGC.

Acknowledgement This work was funded by the State Grid Corporation of China project: Cooperative Simulation of Power Grid and Communication Grid, the National Natural Science Funds 51407030 and China Postdoctoral Science Foundation 121809.

Open Access This article is distributed under the terms of the Creative Commons Attribution 4.0 International License (http:// creativecommons.org/licenses/by/4.0/), which permits unrestricted use, distribution, and reproduction in any medium, provided you give appropriate credit to the original author(s) and the source, provide a link to the Creative Commons license, and indicate if changes were made.

\section{References}

[1] Mohammadi M, Hosseinian SH, Gharehpetian GB (2012) Optimization of hybrid solar energy sources/wind turbine systems integrated to utility grids as microgrid (MG) under pool/bilateral/hybrid electricity market using PSO. Solar Energy 86(1):112-125

[2] Li YW, Nejabatkhah F (2014) Overview of control, integration and energy management of microgrids. J Mod Power Syst Clean Energy 2(3):212-222. doi:10.1007/s40565-014-0063-1

[3] Chen YH, Lu SY, Chang YR et al (2013) Economic analysis and optimal energy management models for microgrid systems: a case study in Taiwan. Appl Energy 103:145-154

[4] Tasdighi M, Ghasemi H, Rahimi-Kian A (2014) Residential microgrid scheduling based on smart meters data and temperature dependent thermal load modeling. IEEE Trans Smart Grid 5(1):349-357

[5] He M, Giesselmann M (2015) Reliability-constrained self-organization and energy management towards a resilient microgrid cluster. In: Proceedings of the 2015 IEEE Power \& Energy Society innovative smart grid technologies conference (ISGT'15), Washington, DC, USA, 18-20 Feb 2015, 5 pp

[6] Sujil A, Agarwal SK, Kumar R (2014) Centralized multi-agent implementation for securing critical loads in PV based microgrid. J Mod Power Syst Clean Energy 2(1):77-86. doi:10.1007/ s40565-014-0047-1 
[7] Arefifar SA, Ordonez M, Mohamed Y (2016) Voltage and current controllability in multi-microgrid smart distribution systems. IEEE Trans Smart Grid (to be published)

[8] Bui DM, Lien KY, Chen SL (2014) Investigate dynamic and transient characteristics for islanded/grid-connected operation modes of microgrid and develop a fast-scalable-adaptable fault protection algorithm. In: Proceedings of the 12th IET international conference on developments in power system protection (DPSP'14), Copenhagen, Danmark, 31 Mar-4 Apr, 2014, 6 pp

[9] Chen CS, Duan SX (2015) Microgrid economic operation considering plug-in hybrid electric vehicles integration. J Mod Power Syst Clean Energy 3(2):221-231. doi:10.1007/s40565015-0116-0

[10] Thale SS, Wandhare RG, Agarwal V (2014) A novel reconfigurable microgrid architecture with renewable energy sources and storage. IEEE Trans Ind Appl 51(2):1805-1816

[11] Radwan AAA, Mohamed YARI (2014) Bidirectional power management in hybrid AC-DC islanded microgrid system. In: Proceedings of the 2014 IEEE PES general meeting, National Harbor, MD, USA, 27-31 Jul 2014, 5 pp

[12] Hamzeh M, Mokhtari H, Karimi H (2013) A decentralized selfadjusting control strategy for reactive power management in an islanded multi-bus MV microgrid. Can J Electr Comput Eng 36(1):18-25

[13] Gong K, Lenz E, Konigorski U (2015) Decentralized frequency control of a DDG-PV microgrid in islanded mode. In: Proceedings of the 23rd mediterranean conference on control and automation (MED'15), Torremolinos, Spain, 16-19 Jun 2015, pp 292-297

[14] Heinbokel B, Kirchhoff H, Dragicevic T et al (2015) Zonal protection of DC swarm microgrids using a novel multi-terminal grid interface with decentralized control. In: Proceedings of the 50th international universities power engineering conference (UPEC'15), Stoke on Trent, UK, 1-4 Sept 2015, 6 pp

[15] Ahn C, Peng H (2013) Decentralized voltage control to minimize distribution power loss of microgrids. IEEE Trans Smart Grid 4(3):1297-1304

[16] Rajagopalan S, Shah D (2011) Distributed averaging in dynamic networks. IEEE J Sel Top Signal Process 5(4):845-854

[17] Wen GH, Duan ZS, Chen GR et al (2014) Consensus tracking of multi-agent systems with Lipschitz-type node dynamics and switching topologies. IEEE Trans Circuits Syst I 61(2):499-511

[18] Zhang W, Liu WX, Wang X et al (2015) Online optimal generation control based on constrained distributed gradient algorithm. IEEE Trans Power Syst 30(1):35-45

[19] Xin HH, Lu ZH, Liu Y et al (2014) A center-free control strategy for the coordination of multiple photovoltaic generators. IEEE Trans Smart Grid 5(3):1262-1269
[20] Yang HM, Yi DX, Zhao JH et al (2013) Distributed optimal dispatch of virtual power plant via limited communication. IEEE Trans Power Syst 28(3):3511-3512

[21] Zhu MH, Martinez S (2012) On distributed convex optimization under inequality and equality constraints. IEEE Trans Autom Control 57(1):151-164

[22] Nedić A, Ozdaglar A (2009) Subgradient methods for saddlepoint problems. J Optim Theory Appl 142(1):205-228

[23] Nedić A, Ozdaglar A, Parrilo PA (2010) Constrained consensus and optimization in multi-agent networks. IEEE Trans Autom Control 55(4):922-938

Jie YU received the B.S. and M.S. degrees in electrical engineering from Southeast University, Nanjing, China, in 1996 and 2000, respectively. She is currently an associate professor in Southeast University after she received $\mathrm{Ph} . \mathrm{D}$. degree in electrical engineering from Southeast University in 2009. Before purchasing her Ph. D., She had worked in NARI Technology Inc. as an electrical engineer from 2000 to 2006. His current research interests include power system optimal dispatch, cyber physical power system, and power market.

Ming NI received the B.S. and Ph.D. degrees in electrical engineering from Southeast University, Nanjing, China, in 1991 and 1996, respectively. He is currently the Special Expert with NARI Technology Inc., Nanjing. Before joining NARI in 2012, he was the Manager of Economic Studies at Midwest ISO, Saint Paul, MN, USA. His current research interests include cyber physical power system, power system stability control, and power system planning.

Yiping JIAO received the B.S. degree at School of IOT, Jiangnan University in 2013, and received the M.S. degree at School of Automation, Southeast University in 2016. He is purchasing Ph.D. in School of Automation, Southeast University for machine learning now. During the postgraduate duration, he worked for Electrical Engineering on distributed computation and optimization. He currently focuses on machine learning and artificial intelligence.

Xiaolong WANG received the B.S. degree at School of Automation, Henan Polytechnic University in 2012, and received the M.S. degree at School of Automation, Southeast University in 2016. He is working for China Aviation Lithium Battery CO., LTD now. 